\title{
On the metallicity distribution of classical Cepheids in the Galactic inner disk $\mathbf{k}^{\star} \star \star$
}

\author{
K. Genovali ${ }^{1}$, B. Lemasle ${ }^{2}$, G. Bono ${ }^{1,3}$, M. Romaniello ${ }^{4}$, F. Primas ${ }^{4}$, M. Fabrizio ${ }^{5}$, R. Buonanno ${ }^{1,5}$, P. François ${ }^{6,7}$, \\ L. Inno ${ }^{1,4}$, C. D. Laney ${ }^{8,9}$, N. Matsunaga ${ }^{10}$, S. Pedicelli ${ }^{1}$, and F. Thévenin ${ }^{11}$ \\ ${ }^{1}$ Dipartimento di Fisica, Università di Roma Tor Vergata, via della Ricerca Scientifica 1, 00133 Rome, Italy \\ e-mail: katia.genovali@roma2.infn.it \\ 2 Astronomical Institute 'Anton Pannekoek', Science Park 904, PO Box 94249, 1090 GE Amsterdam, The Netherlands \\ 3 INAF - Osservatorio Astronomico di Roma, via Frascati 33, Monte Porzio Catone, Rome, Italy \\ ${ }^{4}$ European Southern Observatory, Karl-Schwarzschild-Str. 2, 85748 Garching bei Munchen, Germany \\ 5 INAF - Osservatorio Astronomico di Collurania, via M. Maggini, 64100 Teramo, Italy \\ ${ }^{6}$ GEPI - Observatoire de Paris, 64 Avenue de l'Observatoire, 75014 Paris, France \\ 7 UPJV - Université de Picardie Jules Verne, 80000 Amiens, France \\ ${ }^{8}$ Department of Physics and Astronomy, N283 ESC, Brigham Young University, Provo, UT 84601, USA \\ 9 South African Astronomical Observatory, PO Box 9, Observatory 7935, South Africa \\ 10 Kiso Observatory, Institute of Astronomy, School of Science, The University of Tokyo 10762-30, Mitake, Kiso-machi, Kiso-gun 3 , \\ 97-0101 Nagano, Japan \\ 11 Laboratoire Lagrange, UMR 7293, Université de Nice Sophia-Antipolis, CNRS, Observatoire de la Côte d'Azur, 06300 Nice, \\ France
}

Received 5 April 2013 / Accepted 22 April 2013

\begin{abstract}
We present homogeneous and accurate iron abundances for almost four dozen (47) of Galactic Cepheids using high-spectral resolution $(R \sim 40000)$ high signal-to-noise ratio $(S / N \geq 100)$ optical spectra collected with UVES at VLT. A significant fraction of the sample (32) is located in the inner disk $\left(R_{\mathrm{G}} \leq 6.9 \mathrm{kpc}\right)$ and for half of them we provide new iron abundances. Current findings indicate a steady increase in iron abundance when approaching the innermost regions of the thin disk. The metallicity is super-solar and ranges from 0.2 dex for $R_{\mathrm{G}} \sim 6.5 \mathrm{kpc}$ to 0.4 dex for $R_{\mathrm{G}} \sim 5.5 \mathrm{kpc}$. Moreover, we do not find evidence of correlation between iron abundance and distance from the Galactic plane. We collected similar data available in the literature and ended up with a sample of 420 Cepheids. Current data suggest that the mean metallicity and the metallicity dispersion in the four quadrants of the Galactic disk attain similar values. The first-second quadrants show a more extended metal-poor tail, while the third-fourth quadrants show a more extended metal-rich tail, but the bulk of the sample is at solar iron abundance. Finally, we found a significant difference between the iron abundance of Cepheids located close to the edge of the inner disk $([\mathrm{Fe} / \mathrm{H}] \sim 0.4)$ and young stars located either along the Galactic bar or in the nuclear bulge $([\mathrm{Fe} / \mathrm{H}] \sim 0)$. Thus suggesting that the above regions have had different chemical enrichment histories. The same outcome applies to the metallicity gradient of the Galactic bulge, since mounting empirical evidence indicates that the mean metallicity increases when moving from the outer to the inner bulge regions.
\end{abstract}

Key words. galaxies: individual: Milky Way - galaxies: stellar content - stars: abundances - stars: fundamental parameters stars: variables: Cepheids

\section{Introduction}

Classical Cepheids after the discovery that they are robust distance indicators (Leavitt \& Pickering 1912) have had a widespread use in stellar astrophysics and in cosmology (Freedman \& Madore 2010). They are very popular since they can be adopted as primary distance indicators (Matsunaga et al. 2011a; Storm et al. 2011b; Freedman et al. 2012; Inno et al. 2013; Groenewegen 2013), as fundamental physics laboratory to constrain evolutionary and pulsation properties of intermediatemass stars (Bono et al. 2010; Pietrzyński et al. 2010; Molinaro

* Based on spectra collected with the spectrograph UVES available at the ESO Very Large Telescope (VLT), Cerro Paranal, (081.D-0928(A) PI: S. Pedicelli - 082.D-0901(A) PI: S. Pedicelli).

$\star \star$ Full Table 1 is only available at the CDS

via anonymous ftp to cdsarc.u-strasbg. fr (130.79.128.5) or via http://cdsarc.u-strasbg.fr/viz-bin/qcat?]/A+A/554/A132 et al. 2011; Prada Moroni et al. 2012; Matthews et al. 2012; Neilson et al. 2012) and as stellar tracers of young stars in the thin disk (Andrievsky et al. 2004; Lemasle et al. 2007, 2008; Romaniello et al. 2008; Pedicelli et al. 2009; Luck \& Lambert 2011) and in the Magellanic Clouds (Storm et al. 2011b; Groenewegen 2013; Haschke et al. 2012).

In particular, the iron and the $\alpha$-element abundance gradients across the Galactic disk are fundamental observables to constrain the chemical enrichment of disk stellar populations (Luck et al. 2006; Lemasle et al. 2008; Luck et al. 2011; Luck \& Lambert 2011). They also play a key role in constraining the physical assumptions adopted in chemical evolution models (Portinari \& Chiosi 2000; Chiappini et al. 2001; Cescutti et al. 2007). The most recent theoretical and empirical investigations brought forward three relevant open issues:

Stellar tracers - Empirical evidence indicates that different stellar tracers do provide different slopes. Metallicity 
gradients based on Cepheids provide slopes of the order of -0.05 dex kpc ${ }^{-1}$ (Caputo et al. 2001; Andrievsky et al. 2002b; Luck et al. 2003, 2006; Kovtyukh et al. 2005; Yong et al. 2006; Lemasle et al. 2007, 2008). More recently, Pedicelli et al. (2009) using iron abundances for 265 classical Cepheids - based either on high-resolution spectra or on photometric metallicity indices - and Galactocentric distances ranging from $R_{\mathrm{G}} \sim 5$ to $R_{\mathrm{G}} \sim 17 \mathrm{kpc}$ found an iron gradient of $-0.051 \pm$ $0.004 \mathrm{dex} \mathrm{kpc}{ }^{-1}$. By using an even larger sample of more than 400 classical Cepheids, Luck \& Lambert (2011) found a gradient of $-0.062 \pm 0.002 \mathrm{dex} \mathrm{kpc}^{-1}$. A similar gradient was also found by Friel et al. (2002) by using a sample of 39 open clusters located between the solar circle and $R_{\mathrm{G}} \sim 14 \mathrm{kpc}$,

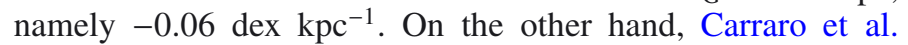
(2007), by using new metallicities for five old open clusters located in the outer disk $\left(12 \leq R_{\mathrm{G}} \leq 21 \mathrm{kpc}\right)$ and the sample adopted by Friel et al. (2002), found a shallower iron gradient: $-0.018 \mathrm{dex} \mathrm{kpc}^{-1}$. The slope of the metallicity gradient based on oxygen abundances of HII regions - with Galactocentric distances ranging from 5 to $15 \mathrm{kpc}$ - is similar to the slope based on Cepheids ( $-0.04 \mathrm{dex} \mathrm{kpc}^{-1}$, Deharveng et al. 2000). The difference between the different tracers might be due to the age difference between the different tracers (young vs. intermediate-age).

Linear slope - By using a sample of 76 open clusters with distances ranging from 6 to $15 \mathrm{kpc}$, it was suggested by Twarog et al. (1997) that a proper fit to the metallicity distribution does require two zones. The inner disk for Galactocentric distances ranging from 6 to $10 \mathrm{kpc}$ and the outer disk for distances larger than $10 \mathrm{kpc}$. This hypothesis was supported by Caputo et al. (2001), Luck et al. (2003), and Andrievsky et al. (2004). More recently, Pedicelli et al. (2009) found for the two zones a slope of $-0.130 \pm 0.015 \mathrm{dex} \mathrm{kpc}^{-1}$ for the inner disk $\left(R_{\mathrm{G}}<8 \mathrm{kpc}\right)$ and a slope of $-0.042 \pm 0.004 \mathrm{dex} \mathrm{kpc}^{-1}$ for the outer disk.

Local inhomogeneities - There is evidence of abundance inhomogeneities across the Galactic quadrants. Pedicelli et al. (2009) found that the iron abundance of the two Cepheid overdensities located in the first and in the third quadrant covers the same metallicity range of the global gradient (see also Luck et al. 2006; Lemasle et al. 2007; Luck et al. 2011).

Azimuthal dependence - In a recent investigation concerning the chemical enrichment of the thin disk based on 398 Cepheids, Luck \& Lambert (2011) did not find evidence of azimuthal dependence. Davies et al. (2009b) suggested that the increase in metallicity in region located between the solar circle and the inner disk might be due to an azimuthal dependence.

The above open issues rely on sizable samples of Galactic classical Cepheids, but they are partially hampered by the fact that abundances based on high-resolution spectra are far from being homogeneous. To overcome these thorny problems, we collected a large sample of high spectral - resolution $(R \sim$ $38000)$ spectra with UVES at VLT for 77 classical Cepheids mainly located in the third and fourth quadrant of the Galactic disk.

\section{Spectroscopic data and iron abundances}

We acquired high-resolution $(R \sim 38000)$ spectra with the UVES spectrograph available at the Nasmyth B focus of UT2/VLT $8 \mathrm{~m}$ telescope (Fig. 1). The spectra were collected at random pulsational phases between 2008 October and 2009 April using the DIC2 $(437+760)$ configuration with blue and red arms operating in parallel. The two arms cover the wavelength intervals $\sim 3750-5000 \AA$ and $~ 5650-7600 / 7660-9460 \AA$ (two chips in the red arm).
Table 1. Fe I and Fe II linelist adopted to estimate iron abundances.

\begin{tabular}{lccc}
\hline \hline$\lambda[\AA]$ & Ion & EP & $\log g f$ \\
\hline 4893.82 & Fe II & 2.83 & -4.45 \\
4917.23 & Fe I & 4.19 & -1.29 \\
4923.93 & Fe II & 2.88 & -1.35 \\
4924.77 & Fe I & 2.28 & -2.24 \\
4932.08 & Fe I & 4.65 & -1.49 \\
$\ldots$ & $\ldots$ & $\ldots$ & $\ldots$ \\
\hline
\end{tabular}

Notes. Iron line list, from left to right the columns display wavelength, ion identification, excitation potential (EP) and $\log g f$ values. The table is available in its entirety via the link to the machine-readable version. A portion is shown here for guidance regarding its form and content.

The complete spectroscopic sample includes 84 spectra for a total of 77 Cepheids. They were collected with exposure times ranging from 80 to $2000 \mathrm{~s}$, while the seeing ranges from 0.6 to 2 arcsec with a mean value of 1.2 arcsec. The quality of the spectra is very good, and indeed the signal-to-noise-ratio $(\mathrm{S} / \mathrm{N})$ is typically better than $\sim 100$ for all the echelle orders. The spectra were reduced using the ESO UVES dedicated pipeline REFLEX v2.1 (Ballester et al. 2011). In this investigation we focus our attention on a sub-sample -47 out of the 77 targets $-\mathrm{a}$ more detailed description of the data reduction will be discussed in a forthcoming paper.

We implemented a semi-automatic procedure to determine the continuum and to fit with a Gaussian the single line profile. The blended lines were fit with the sum of two Gaussians. We adopted the iron linelist provided by Romaniello et al. (2008) and typically we measured the equivalent width (EW) of $\sim 250 \mathrm{Fe}_{\mathrm{I}}$ and $\sim 40 \mathrm{Fe}$ II lines. Some of these lines are heavily blended in our spectra and to overcome thorny problems in the estimate of the mean iron abundance, they were neglected in the estimate of the abundance. For the spectra lacking of useful Fe II (i.e. with EWs smaller than $\sim 150 \AA$ ) we also measured the Fe II lines listed in Pedicelli et al. (2010). The final number of adopted lines is $\sim 230$ for $\mathrm{Fe}$ I and $\sim 55$ for Fe II, respectively (see Table 1).

We updated the atomic data (oscillator strength and excitation potential) of the lines according to the values available in VALD (Vienna Atomic Lines Database ${ }^{1}$, Kupka et al. 2000).

Iron abundances were estimated using MARCS atmospheric models (Gustafsson et al. 2008). Once we estimated the atmospheric parameters and provided a preliminary estimate of the metallicity, we performed an interpolation over a grid of LTE, plane-parallel models by using the dedicated code for abundance determination, fitline, developed by one of the co-authors (François).

For each spectrum we computed the curves of growth, for both neutral and ionized iron together with the plots showing $\left[\mathrm{Fe}_{\mathrm{I}} / \mathrm{H}\right]$ vs. excitation potential and $\left[\mathrm{Fe}_{\mathrm{I}} / \mathrm{H}\right]$ vs. EWs. The process is iterated until a good match between predicted and observed equivalent widths and metallicity is approached.

The effective temperature $-T_{\text {eff }}-$ for individual spectra was independently estimated by using the line depth ratios (LDRs) method (Kovtyukh \& Gorlova 2000). Typically, we measured two dozen LDRs per spectrum. The surface gravity $-\log g-$ is derived by imposing the ionization balance between Fe I and Fe II. The individual micro-turbulent velocities $-v_{t}-$ were estimated by minimizing the $[\mathrm{Fe} / \mathrm{H}] / \mathrm{EW}$ slope, together with a visual inspection of the curves of growth.

1 http://www. astro.uu.se/ vald/php/vald.php 
K. Genovali et al.: Cepheid metallicity distribution in the Galactic inner disk

Table 2. Sample of Galactic Cepheids for which the iron abundance was measured using high-resolution, high signal-to-noise UVES spectra.

\begin{tabular}{|c|c|c|c|c|c|c|c|c|c|}
\hline Name & $\begin{array}{c}\log (P) \\
\text { [days] }\end{array}$ & $\begin{array}{c}\langle J\rangle \\
\text { mag }\end{array}$ & $\begin{array}{l}\langle H\rangle \\
\text { mag }\end{array}$ & $\begin{array}{l}\langle K\rangle \\
\text { mag }\end{array}$ & {$[\mathrm{Fe} / \mathrm{H}]$} & $\begin{array}{c}\mu^{e} \\
\mathrm{mag}\end{array}$ & $\begin{array}{l}R_{\mathrm{G}}{ }^{f} \\
(\mathrm{pc})\end{array}$ & \multicolumn{2}{|c|}{ Notes } \\
\hline V340 Ara & 1.3183 & 7.302 & 6.754 & 6.534 & $0.53 \pm 0.09$ & $12.99 \pm 0.05$ & $4657 \pm 427$ & $\mathrm{~b}^{\dagger}$ & \\
\hline AS Aur & 0.5017 & 9.90 & 9.46 & 9.33 & $0.00 \pm 0.08$ & $13.18 \pm 0.06$ & $12244 \pm 469$ & $\mathrm{c}$ & \\
\hline KN Cen & 1.5321 & 6.439 & 5.769 & 5.474 & $0.55 \pm 0.12$ & $12.51 \pm 0.05$ & $6498 \pm 417$ & $\mathrm{~b}$ & \\
\hline MZ Cen & 1.0151 & 8.44 & 7.87 & 7.61 & $0.27 \pm 0.10$ & $13.01 \pm 0.07$ & $6501 \pm 391$ & $\mathrm{c}$ & \\
\hline OO Cen & 1.1099 & 8.39 & 7.72 & 7.45 & $0.20 \pm 0.06$ & $13.08 \pm 0.07$ & $6025 \pm 389$ & $\mathrm{c}$ & $\#^{d}$ \\
\hline TX Cen & 1.2328 & 7.17 & 6.66 & 6.40 & $0.44 \pm 0.13$ & $12.58 \pm 0.07$ & $6070 \pm 419$ & $\mathrm{c}$ & \# \\
\hline V339 Cen & 0.9762 & 6.354 & 5.877 & 5.690 & $0.06 \pm 0.03$ & $11.07 \pm 0.05$ & $6917 \pm 446$ & $\mathrm{~b}$ & \\
\hline VW Cen & 1.1771 & 7.588 & 7.028 & 6.805 & $0.41 \pm 0.08$ & $12.78 \pm 0.05$ & $6417 \pm 405$ & $\mathrm{~b}$ & \\
\hline RW CMa & 0.7581 & 8.43 & 7.96 & 7.75 & $-0.07 \pm 0.08$ & $12.38 \pm 0.07$ & $10057 \pm 445$ & $\mathrm{c}$ & \# \\
\hline SS CMa & 1.0921 & 7.370 & 6.863 & 6.664 & $0.06 \pm 0.04$ & $12.40 \pm 0.05$ & $9829 \pm 439$ & $\mathrm{~b}$ & \\
\hline TV CMa & 0.6693 & 8.022 & 7.582 & 7.364 & $0.01 \pm 0.07$ & $11.72 \pm 0.05$ & $9575 \pm 447$ & $\mathrm{a}$ & \\
\hline AA Gem & 1.0532 & 7.636 & 7.201 & 7.048 & $-0.08 \pm 0.05$ & $12.74 \pm 0.05$ & $11454 \pm 459$ & $\mathrm{a}$ & \\
\hline BW Gem & 0.4208 & 9.83 & 9.30 & 9.16 & $-0.22 \pm 0.09$ & $12.65 \pm 0.07$ & $11302 \pm 463$ & $\mathrm{c}$ & \\
\hline FT Mon & 0.6843 & 10.400 & 9.937 & 9.754 & $-0.13 \pm 0.08$ & $14.16 \pm 0.05$ & $14344 \pm 468$ & $\mathrm{~b}^{\dagger}$ & \\
\hline SV Mon & 1.1828 & 6.249 & 5.829 & 5.666 & $0.12 \pm 0.08$ & $11.80 \pm 0.05$ & $10070 \pm 453$ & $\mathrm{a}$ & \\
\hline TY Mon & 0.6045 & 9.302 & 8.865 & 8.668 & $0.02 \pm 0.08$ & $12.82 \pm 0.05$ & $11180 \pm 451$ & $\mathrm{a}$ & \\
\hline TZ Mon & 0.8709 & 8.447 & 8.005 & 7.795 & $-0.02 \pm 0.07$ & $12.83 \pm 0.05$ & $11183 \pm 451$ & $\mathrm{a}$ & \\
\hline GU Nor & 0.5382 & 7.61 & 7.17 & 6.97 & $0.08 \pm 0.06$ & $10.90 \pm 0.07$ & $6663 \pm 450$ & $\mathrm{c}$ & \\
\hline IQ Nor & 0.9159 & 6.79 & 6.24 & 6.02 & $0.22 \pm 0.07$ & $11.12 \pm 0.08$ & $6691 \pm 448$ & $\mathrm{c}$ & \# \\
\hline QZ Nor & 0.5782 & 7.085 & 6.748 & 6.614 & $0.19 \pm 0.08$ & $11.53 \pm 0.05$ & $6283 \pm 447$ & $\mathrm{~b}$ & \# \\
\hline RS Nor & 0.7923 & 7.32 & 6.85 & 6.64 & $0.18 \pm 0.08$ & $11.39 \pm 0.07$ & $6385 \pm 449$ & $\mathrm{c}$ & \# \\
\hline SY Nor & 1.1019 & 6.638 & 6.091 & 5.864 & $0.27 \pm 0.10$ & $11.59 \pm 0.05$ & $6286 \pm 446$ & $\mathrm{~b}^{\dagger}$ & \\
\hline TW Nor & 1.0329 & 7.442 & 6.712 & 6.375 & $0.27 \pm 0.10$ & $11.67 \pm 0.05$ & $6160 \pm 447$ & $\mathrm{~b}$ & \\
\hline V340 Nor & 1.0526 & 6.211 & 5.745 & 5.573 & $0.07 \pm 0.07$ & $11.22 \pm 0.05$ & $6483 \pm 449$ & $\mathrm{~b}$ & \\
\hline RS Ori & 0.8789 & 6.398 & 6.020 & 5.860 & $0.11 \pm 0.09$ & $11.00 \pm 0.05$ & $9470 \pm 453$ & $\mathrm{a}$ & \\
\hline BM Pup & 0.8572 & 8.42 & 7.93 & 7.76 & $-0.07 \pm 0.08$ & $12.74 \pm 0.07$ & $9981 \pm 435$ & $\mathrm{c}$ & \# \\
\hline CK Pup & 0.8703 & 10.273 & 9.703 & 9.456 & $-0.12 \pm 0.08$ & $14.37 \pm 0.05$ & $13357 \pm 423$ & $\mathrm{~b}^{\dagger}$ & \# \\
\hline WY Pup & 0.7202 & 8.945 & 8.577 & 8.453 & $-0.10 \pm 0.08$ & $13.09 \pm 0.05$ & $10549 \pm 430$ & $\mathrm{~b}^{\dagger}$ & \# \\
\hline WZ Pup & 0.7013 & 8.71 & 8.33 & 8.20 & $-0.07 \pm 0.06$ & $12.76 \pm 0.07$ & $10123 \pm 437$ & $\mathrm{c}$ & \# \\
\hline KQ Sco & 1.4577 & 5.945 & 5.229 & 4.924 & $0.52 \pm 0.08$ & $11.67 \pm 0.05$ & $5948 \pm 451$ & $\mathrm{~b}$ & \\
\hline RY Sco & 1.3078 & 4.930 & 4.379 & 4.134 & $0.06 \pm 0.02$ & $10.54 \pm 0.05$ & $6663 \pm 453$ & $\mathrm{~b}$ & \\
\hline V470 Sco & 1.2112 & 6.01 & 5.31 & 4.98 & $0.16 \pm 0.06$ & $10.89 \pm 0.07$ & $6461 \pm 454$ & $\mathrm{c}$ & \# \\
\hline EV Sct & 0.4901 & 7.608 & 7.184 & 7.018 & $0.09 \pm 0.07$ & $11.54 \pm 0.05$ & $6135 \pm 449$ & $\mathrm{~b}$ & \\
\hline RU Sct & 1.2945 & 5.891 & 5.287 & 5.034 & $0.16 \pm 0.05$ & $11.35 \pm 0.05$ & $6361 \pm 449$ & $\mathrm{a}$ & \\
\hline UZ Sct & 1.1686 & 7.405 & 6 & 6.461 & $0.45 \pm$ & $12.29 \pm 0.05$ & $5309 \pm 448$ & $\mathrm{a}$ & \\
\hline V367 Sct & 0.7989 & 7.605 & 6.955 & 6.651 & $-0.04 \pm 0.04$ & $11.24 \pm 0.05$ & $6332 \pm 451$ & $\mathrm{~b}$ & \\
\hline X Sct & 0.6230 & 7.378 & 6.952 & 6.768 & $0.12 \pm 0.09$ & $11.00 \pm 0.05$ & $6464 \pm 452$ & $\mathrm{a}$ & \# \\
\hline Z Sct & 1.1106 & 6.949 & 6.477 & 6.263 & $0.18 \pm 0.09$ & $12.08 \pm 0.05$ & $5733 \pm 445$ & $\mathrm{a}$ & \\
\hline AA Ser & 1.2340 & 7.563 & 6.773 & 6.457 & $0.38 \pm 0.21$ & $12.39 \pm 0.05$ & $5572 \pm 437$ & $\mathrm{a}$ & \\
\hline CR Ser & 0.7244 & 7.340 & 6.759 & 6.485 & $0.12 \pm 0.08$ & $10.89 \pm 0.05$ & $6510 \pm 452$ & $\mathrm{a}$ & \# \\
\hline AV Sgr & 1.1879 & 6.878 & 6.089 & 5.730 & $0.35 \pm 0.17$ & $11.49 \pm 0.05$ & $5980 \pm 455$ & $b^{\dagger}$ & \\
\hline AY Sgr & 0.8175 & 7.127 & 6.531 & 6.264 & $0.11 \pm 0.06$ & $10.97 \pm 0.05$ & $6429 \pm 452$ & $\mathrm{a}$ & \# \\
\hline V1954 Sgr & 0.7909 & 7.81 & 7.33 & 7.10 & $0.24 \pm 0.10$ & $11.82 \pm 0.07$ & $5687 \pm 456$ & c & \# \\
\hline V773 Sgr & 0.7596 & 7.52 & 6.70 & 6.35 & $0.11 \pm 0.06$ & $10.65 \pm 0.07$ & $6595 \pm 454$ & $\mathrm{c}$ & \# \\
\hline VY Sgr & 1.1322 & 7.156 & 6.385 & 6.042 & $0.43 \pm 0.14$ & $11.63 \pm 0.05$ & $5862 \pm 453$ & $\mathrm{~b}^{\dagger}$ & \\
\hline WZ Sgr & 1.3394 & 5.255 & 4.752 & 4.536 & $0.35 \pm 0.08$ & $11.10 \pm 0.05$ & $6326 \pm 453$ & $\mathrm{a}$ & \\
\hline XX Sgr & 0.8078 & 6.44 & 5.97 & 5.75 & $-0.07 \pm 0.07$ & $10.55 \pm 0.07$ & $6706 \pm 453$ & $\mathrm{c}$ & \\
\hline
\end{tabular}

Notes. From left to right: target name, period, $J, H, K$ mean magnitudes, iron abundance, distance modulus and Galactocentric distance. Notes on the photometric data: ${ }^{(a)}$ mean magnitudes provided by Monson \& Pierce (2011) transformed into the 2MASS photometric system using the relations given in their Table 1. ${ }^{(b)}$ Mean magnitudes provided by Laney \& Stobie (1992) and Laney (priv. comm.) transformed into the 2MASS photometric system using the relations given by Koen et al. (2007). The objects marked with a ${ }^{(\dagger)}$ (Laney, priv. comm.) do not have a complete coverage of the light-curve (the number of phase points ranges from 4 to 14). ${ }^{(c)}$ Mean magnitudes based on single-epoch measurements from the 2MASS catalogue and the NIR template light curves provided by Soszyński et al. (2005). ${ }^{(d)}$ Classical Cepheids for which the spectroscopic iron abundance is measured for the first time. ${ }^{(e)}$ The weighted mean true distance moduli. The errors account for uncertainties affecting mean magnitudes and for the intrinsic dispersion of the adopted NIR PW relations. ${ }^{(f)}$ The weighted mean Galactocentric distances were estimated assuming $R_{\mathrm{G}}=7.94 \pm 0.37 \pm 0.26 \mathrm{kpc}$ (Groenewegen et al. 2008). The errors account for uncertainties affecting both the solar Galactocentric distance and the heliocentric distances.

The maximum EW value included in the metallicity determination varies according to the metallicity itself and on the atmospheric parameters of the star. For a significant fraction of our spectra we were able to use only relatively weak lines $(E W<120 \mathrm{~m} \AA)$ located along the linear part of the curve of growth. In a few cases the spectra were lacking of a significant number of weak lines (less than two Fe II lines), therefore, we included in the analysis also lines with $E W \leq 180 \mathrm{~m} \AA$. The latters cause a mild increase in the uncertainties affecting the atmospheric parameters. 


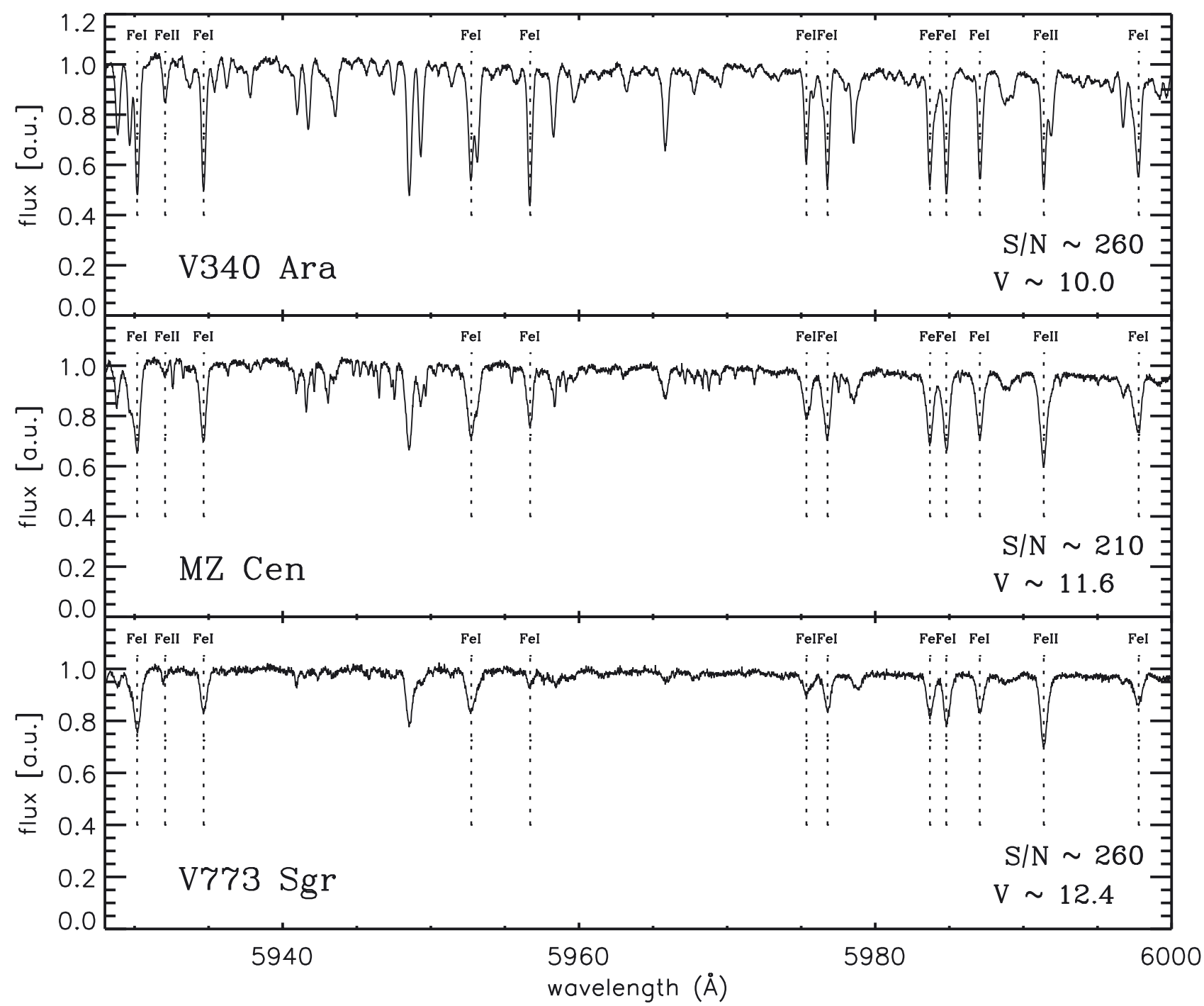

Fig. 1. Selected high-resolution $(R \sim 38000)$ UVES spectra of Galactic Cepheids in our sample. From top to bottom are plotted the spectra of V340 Ara $([\mathrm{Fe} / \mathrm{H}]=0.53 \pm 0.09)$, MZ Cen $([\mathrm{Fe} / \mathrm{H}]=0.27 \pm 0.10)$ and $\mathrm{V} 773 \mathrm{Sgr}([\mathrm{Fe} / \mathrm{H}]=0.11 \pm 0.06)$. The apparent visual magnitude and the $\mathrm{S} / \mathrm{N}$ in the spectral range $\lambda \sim 5650-7500 \AA$ (red arm) are also labeled. The vertical dashed lines display selected Fe I $(\lambda \lambda 5930.17,5934.66$, $5952.73,5956.7,5975.35,5976.78,5983.69,5984.79,5987.05,5997.78 \AA)$ and Fe II ( $\lambda \lambda 5932.06$ and $5991.37 \AA$ ) lines included in our abundance analysis (see Table 1 and Romaniello et al. 2008).

The mean iron abundances given in Table 2 are the weighted mean of $[\mathrm{Fe} \mathrm{I} / \mathrm{H}]$ and $[\mathrm{Fe} \mathrm{I} / \mathrm{H}]$ lines. The errors listed in column six are the standard deviations of the weighted mean, i.e. $\sigma_{\mathrm{Fe}}=$ $\sqrt{\sigma_{\mathrm{FeI}}^{2}+\sigma_{\mathrm{Fe} \mathrm{II}}^{2}}$, where $\sigma_{\mathrm{Fe} \mathrm{I}}$ and $\sigma_{\mathrm{Fe} \mathrm{II}}$ are the standard deviations of $\left[\mathrm{Fe}_{\mathrm{I}} / \mathrm{H}\right]$ and $[\mathrm{Fe} \mathrm{II} / \mathrm{H}]$ lines. The iron abundances were estimated assuming the solar iron abundance provided by Grevesse et al. (1996), i.e. $\mathrm{A}(\mathrm{Fe})_{\odot}=7.50$.

\section{Photometric data and distance estimates}

To provide a homogeneous sample of Galactocentric distances $\left(R_{\mathrm{G}}\right)$, we adopted near-infrared (NIR) photometric data together with the reddening-free Period-Wesenheit relations in $J, H, K_{\mathrm{s}}$ bands provided by Inno et al. (2013). We estimated individual distances for a significant fraction of Galactic Cepheids (Genovali et al., in prep.). The NIR photometric catalogs we adopted are the following:

SAAO sample - The bulk of this data set comes from Laney $\&$ Stobie 1992. The individual measurements provide an accurate coverage of the light curve and the typical accuracy of mean $J, H, K_{\mathrm{s}}$ magnitudes is at the millimag level. This data set was complemented with new NIR measurements (Laney, priv. comm.) and includes objects with either complete or partial coverage of the light curve. For the latter group the number of phase points along the light curve ranges from four to 14. Their mean magnitudes were estimated using a spline. The SAAO NIR magnitudes were transformed into the 2MASS photometric system using the transformations provided by Koen et al. (2007).

Monson $\mathcal{E}$ Pierce sample - In a recent investigation Monson \& Pierce (2011) published accurate NIR magnitudes for 131 northern hemisphere Cepheids. Their NIR mean magnitudes were transformed into the 2MASS photometric system using the transformations provided by the same authors. The measurements properly cover the entire pulsation cycle and their typical accuracy is better than $0.01 \mathrm{mag}$.

2MASS sample - The above samples were complemented with 2MASS single-epoch observations (Skrutskie et al. 2006). The mean NIR magnitude of fundamental (FU) Cepheids were estimated by using single-epoch photometry and the light-curve template provided by Soszyński et al. (2005). The pulsation properties required to apply the template (epoch of maximum, optical amplitudes, periods) come from General Catalog of 
Variable Stars (GCVS ${ }^{2}$; Samus et al. 2009). The error on the mean NIR magnitudes was estimated as $\sigma_{J, H, K_{\mathrm{s}}}^{2}=\sigma_{\text {phot }}^{2}+\sigma_{\text {temp }}^{2}$, where $\sigma_{\text {phot }}$ is the intrinsic photometric error - typically of the order of $0.03 \mathrm{mag}$ for the Cepheids in the current sample - and $\sigma_{\text {temp }}=0.03 \mathrm{mag}$ is the uncertainty associated with the intrinsic scatter of the template ${ }^{3}$.

For the objects in common in the three different samples, we adopted among them the most accurate. The individual distances were estimated as weighted mean of the three different distances obtained by adopting the NIR $(H, J-H ; K, J-K ; K, H-K)$ Period-Wesenheit (PW) relations provided by Inno et al. (2013). The individual distance moduli are listed in Col. 7 of Table 2 with their uncertainties. The Galactocentric distances listed in Col. 8 of Table 2 were estimated assuming a solar Galactocentric distance of $7.94 \pm 0.37 \pm 0.26 \mathrm{kpc}$ (Groenewegen et al. 2008; Matsunaga et al. 2013, and references therein). The final error on $R_{\mathrm{G}}$ accounts for errors affecting both the solar Galactocentric distance and the heliocentric distances.

We compared current individual distances based on NIR PW relation with individual distances estimated using two different flavors of the IRSB method and we found that the mean difference over the entire sample ranges from $8 \pm 2 \%$ (Groenewegen 2013, 130 stars in common) to $3 \pm 2 \%$ (Storm et al. 2011a, $\sim 80$ stars in common).

\section{The metallicity gradient}

To further constrain the nature of the metallicity gradient of the Galactic disk, in Fig. 2 we plotted both new (16, red circles) and updated (31, blue circles) Cepheid iron abundances as a function of the Galactocentric distances $\left(R_{\mathrm{G}}\right)$. Together with the current sample, we also included iron abundance for Galactic Cepheids estimated by our group using the same approach and similar data (Lemasle et al. 2007, 2008; 57, [LEM], green circles; Romaniello et al. 2008; 16, [ROM], yellow circles). Moreover, we also included Cepheid iron abundances available in the literature (Sziládi et al. 2007; Luck et al. 2011; Luck \& Lambert 2011; 300, [LUCII, LUCIII], black circles). We ended up with a sample of 420 Cepheids. The different sets of abundances were re-scaled to the same metallicity scale ${ }^{4}$.

The current sample has two indisputable advantages: a) iron abundances are based on high-resolution spectra and they were rescaled to the same solar abundance adopted in Sect. 2; b) distance estimates are homogeneous and based on PW relations that are independent of reddening uncertainties and minimally affected by metallicity dependence (Inno et al. 2013).

Data plotted in Fig. 2 show that the metallicity gradient shows a large intrinsic dispersion around the solar circle with the possible occurrence either of a change in the slope or of a shoulder for $7 \leq R_{\mathrm{G}} \leq 10 \mathrm{kpc}$ (Andrievsky et al. 2004). This finding supports previous results based on open clusters and on Cepheids obtained by Twarog et al. (1997) and by Caputo et al. (2001). However, a quantitative analysis of this issue does require a large sample of homogeneous and accurate iron abundance across the solar circle. The metallicity gradient also shows an increase in

\footnotetext{
2 http://www.sai.msu.su/gcvs/gcvs/index.htm

Current sample includes only two first overtones - EV Sct, QZ Nor and their mean magnitudes were provided by Laney \& Stobie (1992).

4 The difference in iron abundance among the different samples are: $\Delta[\mathrm{Fe} / \mathrm{H}](\mathrm{ROM}-\mathrm{us})=-0.06 \pm 0.10, \Delta[\mathrm{Fe} / \mathrm{H}](\mathrm{LEM}-$ us $)=-0.11 \pm 0.09$, $\Delta[\mathrm{Fe} / \mathrm{H}](\mathrm{LUCII}-\mathrm{us})=-0.07 \pm 0.13$ and $\Delta[\mathrm{Fe} / \mathrm{H}](\mathrm{LUCIII}-$ us $)=0.04 \pm$ 0.08. The difference with the seven Cepheids by Sziládi et al. (2007) was not estimated, since we only have one object in common.
}

the intrinsic dispersion in the outer disk $\left(R_{\mathrm{G}} \geq 13 \mathrm{kpc}, \sigma(\mathrm{Fe} / \mathrm{H}) \sim\right.$ $0.15)$. This finding supports previous results by Pedicelli et al. (2009) and by Luck et al. (2011). There is also a mild evidence of a flattening, but the number of outer disk Cepheids is too limited to constrain the possible occurrence of a change in the slope (Carraro et al. 2007). Moreover, Cepheids located in the inner disk $\left(R_{\mathrm{G}} \leq 6.9 \mathrm{kpc}\right)$ show a well defined steepening in the gradient (Andrievsky et al. 2002a; Pedicelli et al. 2010) and a steady trend concerning the intrinsic dispersion $(\sigma(\mathrm{Fe} / \mathrm{H}) \sim 0.1)$. It is worth mentioning that the current sample increases by $20 \%$ the number of inner disk Cepheids with spectroscopic abundances and together with the updated iron abundances we are providing homogeneous abundances for the $55 \%$ of inner disk Cepheids.

The zoom on the region located between the Galactic center and the inner disk (bottom panel of Fig. 2) shows that Cepheids located in the inner disk attain super-solar iron abundances. The trend is quite clear down to $R_{\mathrm{G}} \sim 5 \mathrm{kpc}$, but becomes less clear towards the edge of the inner disk. Indeed, only two Cepheids in our sample have smaller Galactocentric distances. Current findings support previous results by Andrievsky et al. (2002a), Pedicelli et al. (2009) and by Luck et al. (2011).

Although current knowledge of the innermost components of the Galactic spheroid - nuclear bulge, Galactic bulge, inner disk - is quite solid (Brand \& Blitz 1993; Honma \& Sofue 1997; Reid et al. 2009), we still lack quantitative constrains on their kinematic structure and on their chemical enrichment history. In particular, current predictions suggest that a presence of a bar-like structure is crucial to support the high star formation rate of the nuclear bulge (Yusef-Zadeh et al. 2009; Davies et al. 2009a; Matsunaga et al. 2011b). As a matter of fact, it is the barlike structure to drag the gas and the molecular clouds from the inner disk into the nuclear bulge (Athanassoula 1992; Kim et al. 2011).

To further constrain this crucial issue we plotted in Fig. 2 the iron abundances for young stars located in the nuclear bulge. The red cross shows the mean iron abundance of two luminous blue variables (LBVs) in the Quintuplet cluster provided by Najarro et al. (2009). The green triangle shows the mean iron abundance of a dozen nitrogen Wolf-Rayet stars (WNLh) in the Arches cluster provided by Martins et al. (2008), while the green square marks the iron abundance of two field red supergiants (RSGs) of the nuclear bulge provided by Davies et al. (2009a). The main outcome of these investigations is that the typical iron abundance of both cluster and field young stellar tracers is solar. This empirical evidence is further supported by recent spectroscopic measurements provided by Davies et al. (2009b) for two Scutum RGS clusters (14 and 12 stars) located at the near end of the Galactic bar (see magenta diamonds in Fig. 2). They found that both of them have sub-solar iron abundance $(-0.2-0.3 \mathrm{dex})$.

The above findings indicate that the iron abundance of stellar tracers located along the Galactic bar and in the nuclear bulge are more metal-poor than classical Cepheids in the inner disk. This appears as a solid evidence, since the difference in age among the different tracers is minimal, i.e. from a few Myr to a few tens of Myr. Thus suggesting that the mechanism(s) feeding of gas the bar and the nuclear bulge might be more complex than currently assumed.

It has also been suggested that the nuclear bulge could have been formed by the Galactic bulge (Morris \& Serabyn 1996; Davies et al. 2009a,b). To further constrain this fundamental issue, we plotted in Fig. 2 the mean metallicity of four Galactic bulge fields recently provided by Zoccali et al. (2008; $\left.l=0^{\circ}, b=-4^{\circ},-6^{\circ},-12^{\circ}\right)$ and by Uttenthaler et al. (2012; $l=0^{\circ}, b=-10^{\circ}$ ). By using high-resolution spectra for a 

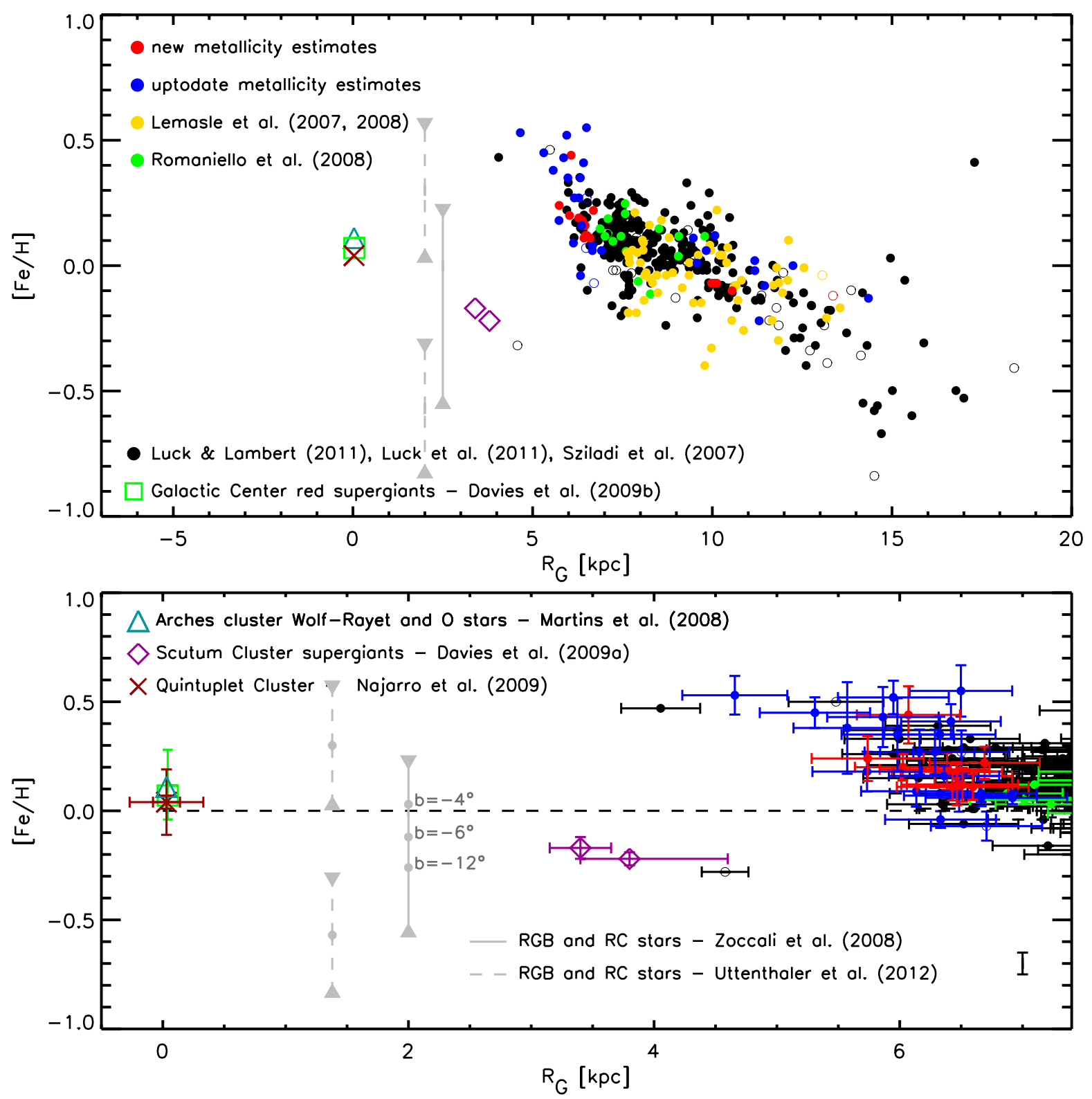

Fig. 2. Top - Iron abundance of Galactic classical Cepheids versus Galactocentric distance. Spectroscopic measurements based on different data sets are plotted with different colors. New iron abundances based on current UVES spectra (16, red), update iron abundances based on UVES spectra (31, blue); iron abundances provided by our group: Lemasle et al. (2008), Lemasle et al. (2007) (57, yellow), Romaniello et al. (2008) (16, green); iron abundances available in the literature: Luck et al. (2011), Luck \& Lambert (2011), and Sziládi et al. (2007) (300, black). Cepheids that according to the General Catalog of Variable Stars (Samus et al. 2009) are candidate classical Cepheids were plotted with open circles. The vertical gray bars located at arbitrary Galactocentric distances $-R_{\mathrm{G}} \sim 2 \mathrm{kpc}-$ show the mean $( \pm 1 \sigma)$ iron abundance for Galactic bulge stars: the solid line is located at the mean metallicity of the sample provided by Zoccali et al. (2008); the dashed lines are located at the mean values $( \pm 1 \sigma)$ found by Uttenthaler et al. (2012). The range in metallicity is $1 \sigma$. The green square marks the iron abundance of the two red supergiants in the Galactic center measured by Davies et al. (2009a), while the magenta diamonds the 26 red supergiants in the Scutum Cluster measured by Davies et al. (2009b), the red cross the two luminous blue variables (LBVs) in the Quintuplet cluster (Najarro et al. 2009) and the light-blue triangle three Wolf-Rayet and two O-type stars in the Arches cluster (Martins et al. 2008). Bottom - Same as the top, but for Galactocentric distances smaller than the solar circle $\left(R_{\mathrm{G}} \leq 7.5 \mathrm{kpc}\right)$. The bars on individual Cepheids display the uncertainty on both iron abundance and distance. The vertical black bar on the bottom right corner shows the mean uncertainty on Luck \& Lambert (2011) and Romaniello et al. (2008) iron abundances. The gray vertical lines are the same of the top panel; the filled circles on the solid line display the mean iron abundance of the bulge fields located at $b=-4^{\circ},(\langle[\mathrm{Fe} / \mathrm{H}]\rangle=+0.03 \pm 0.38), b=-6^{\circ},(\langle[\mathrm{Fe} / \mathrm{H}]\rangle=-0.12 \pm 0.35)$, and at $b=-12^{\circ},(\langle[\mathrm{Fe} / \mathrm{H}]\rangle=-0.26 \pm 0.40)$ observed by Zoccali et al. (2008). The filled circles on the dashed lines display the mean metallicities provided by Uttenthaler et al. (2012) for stars located at $b=-10^{\circ}$, $(\langle[\mathrm{Fe} / \mathrm{H}]\rangle=-0.57 \pm 0.27$ and $\langle[\mathrm{Fe} / \mathrm{H}]\rangle=+0.30 \pm 0.28)$.

large sample of field Red Giant and Red Clump stars (521), Zoccali et al. $(2003,2008)$ and Hill et al. (2011) found that the metallicity distribution of Galactic bulge stars shows a broad main peak $[\mathrm{Fe} / \mathrm{H}] \approx-0.15$ and a large spread in metallicity with iron abundances ranging ( $1 \sigma$ variation) from metal-rich
$([\mathrm{Fe} / \mathrm{H}] \sim-0.55)$ to super solar $([\mathrm{Fe} / \mathrm{H}] \sim+0.25$, see the vertical solid gray line in Fig. 2). The same outcome applies to the sample of $383 \mathrm{RC}$ and RG stars measured by Uttenthaler et al. (2012), with iron abundances ranging ( $1 \sigma$ variation) from $[\mathrm{Fe} / \mathrm{H}] \sim-0.85$ to $\sim 0.17$ (see the vertical dashed gray line in 
K. Genovali et al.: Cepheid metallicity distribution in the Galactic inner disk
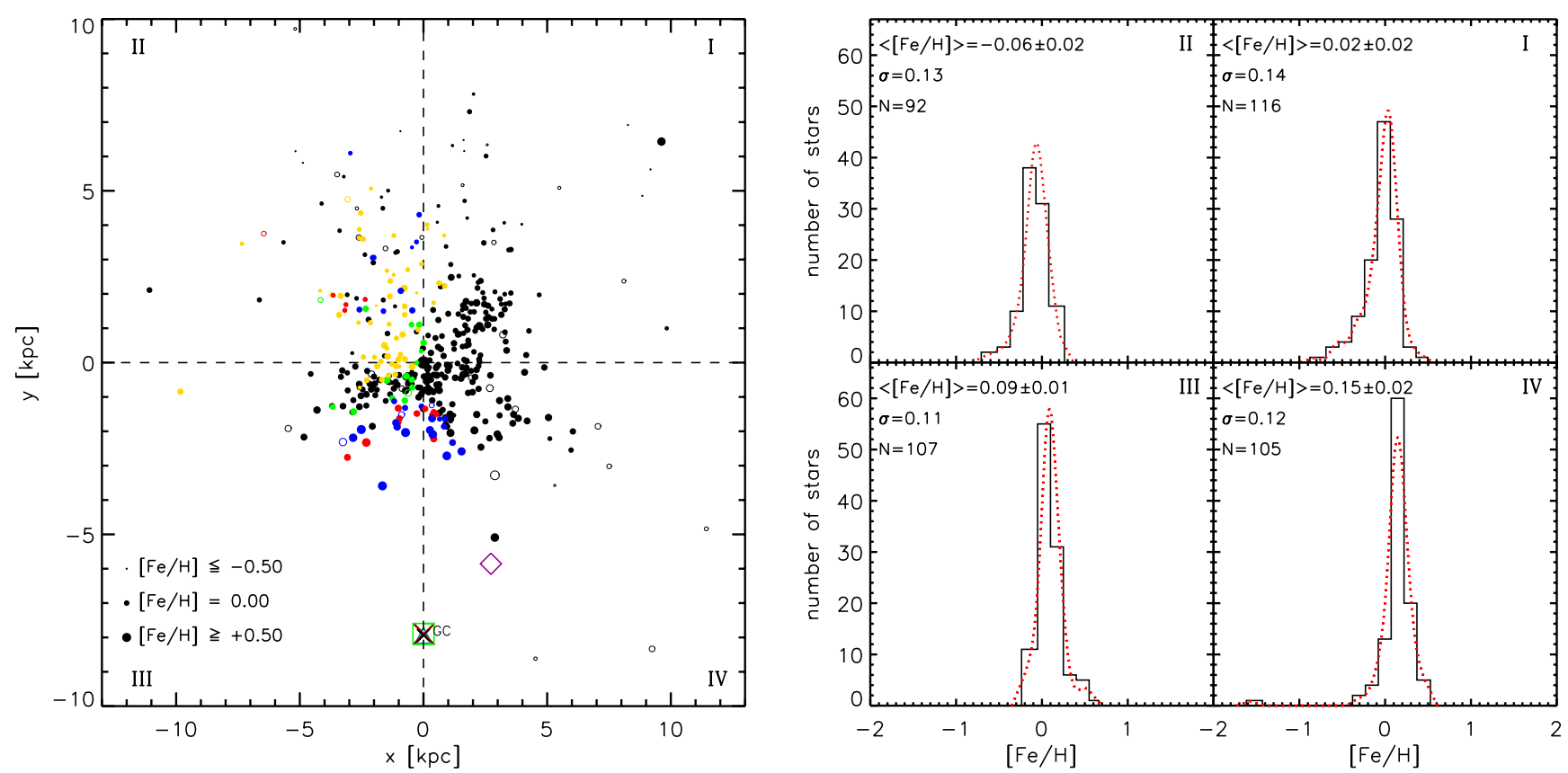

Fig. 3. Left - Distribution onto the Galactic plane of Cepheids with accurate iron abundances. Symbols and colors are the same as in Fig. 2. The size of the circles scales with their iron abundances (see labeled values). The bulge fields were not plotted, since we lack individual distances. The dashed lines display the position of the Sun, while the cross the position of the Galactic center. Right - Metallicity distribution of the Cepheids in the four quadrants. The solid black line shows the histogram (bin size $=0.15 \mathrm{dex}$ ). The dotted red line shows the smoothed metallicity distribution estimated running a Gaussian kernel with fixed $\sigma=0.1$ dex. The adopted dispersion accounts for errors on abundances and uncertainties in the different samples. The number of Cepheids, the mean and the $\sigma$ of the Gaussian fit are also labeled.

Fig. 2). Unfortunately, we lack detailed information concerning the Galactocentric distances of the above bulge fields, however, plain geometrical arguments concerning their Galactic coordinate indicate that, on average, the metallicity gradient is steadily increasing when moving from the outer to the inner edge of the Galactic bulge (Ness et al. 2013). This appears a solid finding, since they adopted old and intermediate-age stellar tracers, i.e. the typical stellar populations of the Galactic bulge.

Once again, the metallicity distributions in the inner disk, in the Galactic bulge, in the nuclear bulge and in the Galactic bar display different trends. Plain leading arguments based on the extrapolation of the inner disk metallicity gradient would imply super-solar iron abundances approaching the Galactic center (Davies et al. 2009a). This discrepancy was already noted in the literature and Cunha et al. (2007) suggested that the slope of the metallicity gradient should become shallower for Galactocentric distances smaller than $5 \mathrm{kpc}$. On the other hand, current findings indicate that young stellar tracers attain super-solar iron abundances approaching the inner edge of the thin disk.

To further constrain the metallicity distribution across the thin disk we also investigated the distribution of Galactic Cepheids onto the Galactic plane. The right panel of Fig. 3 shows the same objects plotted in Fig. 2, but the symbol size scales with the iron abundance (see labeled values). Current Cepheid sample (47) allowed us to improve the spatial sampling in the third and in the fourth quadrant. We now have roughly one hundred Cepheids per quadrant and their mean metallicities and metallicity dispersions attain similar values. There is evidence of an increase in the mean metallicity when moving from the first-second to the third-fourth quadrants, but the difference is on average smaller than 0.2 dex. To provide more quantitative constraints on the recent chemical enrichment history across the thin disk, we estimated the metallicity distribution of the four quadrants. Data plotted in the right panel of Fig. 3 show both the histograms (black line) and the smoothed distributions (red dotted line) of the iron abundances. The latter was estimated by running a Gaussian kernel with a mean $\sigma$ of 0.1 dex. The adopted $\sigma$ accounts for errors on individual abundances and for uncertainties among different samples. The mean and the $\sigma$ of the Gaussian fit of the smoothed distributions are labeled. Data plotted in the right panel show, once again, that the difference is on average smaller than 0.2 dex, moreover, the $\sigma$ attain very similar values.

The above findings indicate that the chemical enrichment across the Galactic disk has been quite homogeneous during the last $\approx 100$ Myr. There is evidence of a more extended metalpoor tail in the first-second quadrant and a more metal-rich tail in the third-fourth quadrant, but the bulk of the sample is at solar iron abundance. There is a mild evidence that the metallicity distribution is probably approaching a plateau toward the shortest Galactocentric distances $\left(R_{\mathrm{G}} \leq 5.5 \mathrm{kpc},[\mathrm{Fe} / \mathrm{H}] \approx 0.4\right)$. However, this preliminary evidence is hampered by the limited number of Cepheids located close to the edge of the inner disk and by the lack of firm empirical estimates of the transition between the disk and the bulge.

In their recent investigation concerning the metallicity distribution of the Galactic bar and of the nuclear bulge, Davies et al. (2009b) suggested that the increase in the mean metal abundance of inner disk Cepheids might be due to azimuthal variations. To constrain a possible change in the azimuthal direction, Fig. 4 shows the distance from the Galactic plane of entire Cepheid sample versus Galactocentric radius. Note that the Cepheid distribution shows a shift compared with the Galactic plane similar to the shift originally found by Kraft \& Schmidt (1964) and by Fernie (1995), i.e. $\Delta Z=-43 \pm 13$ pc (420 stars). It is worth mentioning that the distribution of azimuthal distances remain roughly constant up to ten $\mathrm{kpc}$. At larger Galactocentric 


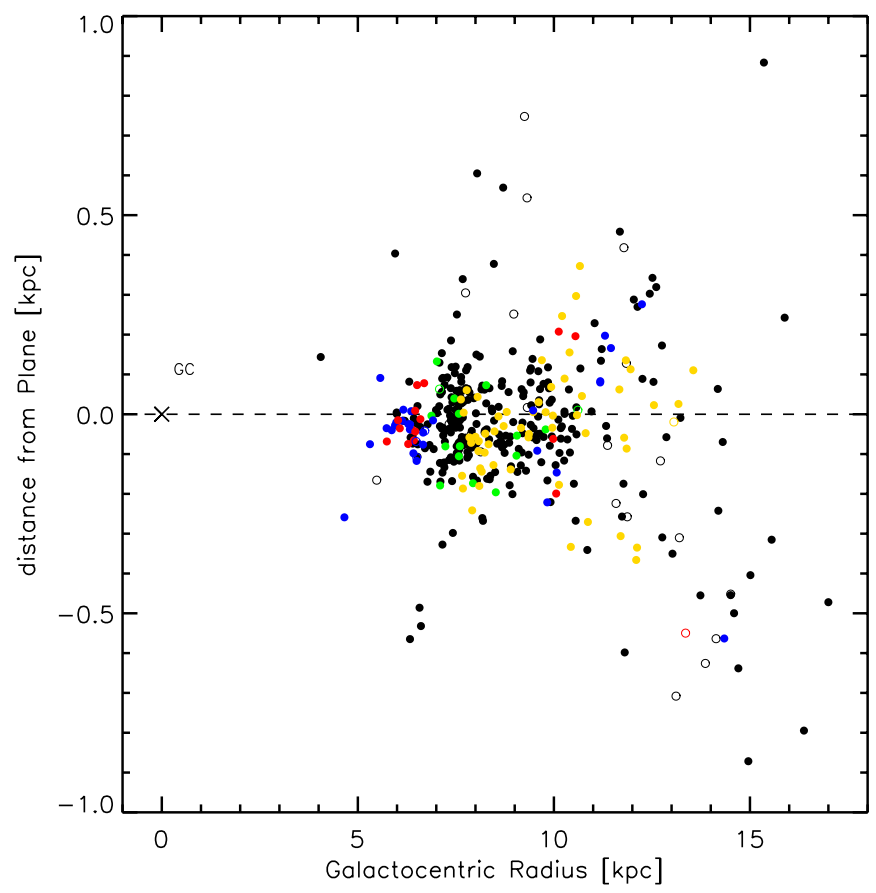

Fig. 4. Distance from the Galactic plane of Galactic Cepheids with accurate iron abundances versus Galactocentric distance. Symbols and colors are the same as in Fig. 2, the black cross marks the Galactic center.

distances the dispersion increases and shows a well defined departure for $R_{\mathrm{G}}$ larger than $13 \mathrm{kpc}$. To constrain a possible metallicity dependence, we split the sample in three sub-samples according to the metallicity and we found that the difference in azimuthal distance among them is smaller than $1 \sigma$.

\section{Conclusions and final remarks}

We performed accurate measurements of iron abundances for 47 Galactic Cepheids using high-resolution, high S/N spectra collected with UVES at VLT. The new sample includes 32 out of the 58 classical Cepheids located in the inner disk $\left(R_{\mathrm{G}} \leq 6.9 \mathrm{kpc}\right)$ for which are available accurate iron abundances.

We found that the metallicity distribution of the inner disk Cepheids is super-solar, and ranges from $\sim 0.2 \operatorname{dex}\left(R_{\mathrm{G}} \approx\right.$ $6.5 \mathrm{kpc})$ to $\sim 0.4 \mathrm{dex}\left(R_{\mathrm{G}} \approx 5.5 \mathrm{kpc}\right)$. Current finding supports previous results (Pedicelli et al. 2009; Luck \& Lambert 2011) concerning a steady increase in metallicity when approaching the innermost disk regions. The new sample allowed us to double the number of Cepheids in the third and in the fourth quadrants when compared with Pedicelli et al. (2009). We found that the difference in the mean metallicity is smaller than 0.2 dex and the metallicity dispersion attain quite similar values in the four quadrants. The metallicity appears to approach a plateau value close to the edge of the inner disk, but this evidence is hampered by the limited number of known Cepheids with $R_{\mathrm{G}} \lesssim 5 \mathrm{kpc}$. In this context, it is worth mentioning that the current sample includes at least the $80 \%$ of the known classical Cepheids located in the inner disk $(62+7$ candidates, Genovali et al., in prep.).

Moreover, we do not find evidence of an azimuthal variation in the Cepheid metallicity distribution. This finding supports previous results by Luck \& Lambert (2011). The Cepheids close to the edge of the inner disk $\left(R_{\mathrm{G}} \approx 4 \mathrm{kpc}\right)$ are significantly more metal-rich than young stellar tracers located along the Galactic bar and in the nuclear bulge. The difference is of the order of 0.5 dex, since Cepheids attain iron abundances of $0.4-0.5$ dex while LBVs and red supergiants have either solar or sub-solar metallicities. This evidence indicates that young stars at the inner edge and at the Galactic bar/nuclear bulge formed from material far from being homogeneous concerning the chemical enrichment.

Theoretical (Athanassoula 1992; Friedli \& Benz 1995) and empirical (Zaritsky et al. 1994; Allard et al. 2006; Zánmar Sánchez et al. 2008) investigations indicate that the abundance gradient in barred galaxies is shallower than in unbarred galaxies. The typical explanation for this trend is that the bar is dragging gas from the inner disk into the nuclear bulge (Kim et al. 2011). The pileup of the new fresh material triggers an ongoing star formation activity till the dynamical stability of the bar. However, there is mounting evidence that in our galaxy the formation and evolution of the above components might have been more complex (Ness et al. 2012). This evidence is further supported by the fact that we are using either massive or intermediate-mass stars (Supergiants, LBVs, Cepheids) and their evolutionary lifetime is typically shorter than $100 \mathrm{Myr}$. This indicates that the bar might not be the main culprit in shaping the metallicity gradient between the inner disk and the nuclear bulge/Galactic bar. Indeed, recent numerical simulations suggest that the timescale within which the radial motion of the gas smooths the actual abundance gradient is of the order of a few hundred Myr (Merlin \& Chiosi 2007). In this timescale part of the azimuthal variations observed across the Galactic disk might be caused by changes in the abundance patterns between the spiral arms and the inter-arm regions (Kim et al. 2011) and by the clumpiness of the star formation episodes. However, the kinematics of the above stellar tracers is quite limited, since they evolve in situ. This working hypothesis is supported by the minimal dependence of the metallicity distribution from the azimuthal distance.

The hypothesis suggested by Davies et al. (2009a) that the wind of metal-intermediate bulge stars might mix with metalrich gas present along the bar and the nuclear bulge to produce a chemical mixture close to solar appears also very promising. However, recent spectroscopic investigations support the evidence of a metallicity distribution that is steadily increasing when moving from the outer to the inner edge of the Galactic bulge (Zoccali et al. 2008; Uttenthaler et al. 2012). Moreover, we still lack firm empirical evidence on how the winds of bulge stars might fall into the nuclear bulge.

Finally, it is worth mentioning that the infall of metal-poor gas in the nuclear bulge appears an even more plausible channel to explain current abundance patterns (Wakker et al. 1999; Lubowich et al. 2000). This is the so-called biased infall scenario (Chiappini et al. 1999) in which the infall of gas takes place more rapidly in the innermost than in the outermost regions (inside-out disk formation).

The abundance pattern of $\alpha$-elements is even more puzzling, since accurate measurements indicate solar abundances, and therefore consistent with typical thin disk stars (Davies et al. 2009b). However, different tracers (B-type stars, red supergiants, classical Cepheids, HII regions) do provide slightly different mean values, suggesting a broad distribution (Davies et al. 2009a, and references therein).

A more quantitative understanding of the above phenomena is important not only to trace the chemical enrichment of the innermost Galaxy regions, but also for its impact on the formation and the evolution of classical bulges and 
pseudo-bulges (Kormendy \& Kennicutt 2004; Kormendy et al. 2009; Matsunaga et al. 2011b; Freeman et al. 2013).

This is the sixth paper in a series focused on the spectroscopic abundances of Galactic Cepheids and together with similar investigations available in the literature we are approaching a complete spectroscopic census of known Galactic Cepheids. However, the current sample is severely affected by an observational bias both in the inner and in the outer disk direction. The new ground-based optical (Large Synoptic Survey Telescope, Chang et al. 2013) and NIR (IRSF/SIRIUS, Nishiyama 2005) photometric surveys together with Gaia will fill this gap.

Acknowledgements. It is a pleasure to thank the referee, R. Earle Luck, for his positive opinion concerning the content and the cut of our paper. This work was partially supported by PRIN-INAF 2011 "Tracing the formation and evolution of the Galactic halo with VST" (PI: M. Marconi) and by PRINMIUR (2010LY5N2T) "Chemical and dynamical evolution of the Milky Way and Local Group galaxies" (PI: F. Matteucci). G.B., K.G. and M.F. thank ESO for support as science visitors. This publication makes use of data products from the Two Micron All Sky Survey, which is a joint project of the University of Massachusetts and the Infrared Processing and Analysis Center/California Institute of Technology, funded by the National Aeronautics and Space Administration and the National Science Foundation. During the writing of this manuscript we learnt that A.N. Cox passed away. We dedicate this manuscript to his memory. His many contributions to the field of stellar pulsation, his steady support to young researchers and his captivating enthusiasm will be greatly missed.

\section{References}

Allard, E. L., Knapen, J. H., Peletier, R. F., \& Sarzi, M. 2006, MNRAS, 371, 1087

Andrievsky, S. M., Bersier, D., Kovtyukh, V. V., et al. 2002a, A\&A, 384, 140

Andrievsky, S. M., Kovtyukh, V. V., Luck, R. E., et al. 2002b, A\&A, 392, 491

Andrievsky, S. M., Luck, R. E., Martin, P., \& Lépine, J. R. D. 2004, A\&A, 413, 159

Athanassoula, E. 1992, MNRAS, 259, 345

Ballester, P., Bramich, D., Forchi, V., et al. 2011, Astronomical Data Analysis Software and Systems XX, 442, 261

Bono, G., Caputo, F., Marconi, M., \& Musella, I. 2010, ApJ, 715, 277

Brand, J., \& Blitz, L. 1993, A\&A, 275, 67

Caputo, F., Marconi, M., Musella, I., \& Pont, F. 2001, A\&A, 372, 544

Carraro, G., Geisler, D., Villanova, S., Frinchaboy, P. M., \& Majewski, S. R. 2007, A\&A, 476, 217

Cescutti, G., Matteucci, F., François, P., \& Chiappini, C. 2007, A\&A, 462, 943

Chang, C., Kahn, S. M., Jernigan, J. G., et al. 2013, MNRAS, 428, 2695

Chiappini, C., Matteucci, F., Beers, T. C., \& Nomoto, K. 1999, ApJ, 515, 226

Chiappini, C., Matteucci, F., \& Romano, D. 2001, ApJ, 554, 1044

Cunha, K., Sellgren, K., Smith, V. V., et al. 2007, ApJ, 669, 1011

Davies, B., Origlia, L., Kudritzki, R.-P., et al. 2009a, ApJ, 694, 46

Davies, B., Origlia, L., Kudritzki, R.-P., et al. 2009b, ApJ, 696, 2014

Davies, B., de La Fuente, D., Najarro, F., et al. 2012, MNRAS, 419, 1860

Deharveng, L., Peña, M., Caplan, J., \& Costero, R. 2000, MNRAS, 311, 329

Fernie, J. D. 1995, IAU Colloq. 155, Astrophysical Applications of Stellar Pulsation, ASP Conf. Ser., 83, 155

Freedman, W. L., \& Madore, B. F. 2010, ARA\&A, 48, 673

Freedman, W. L., Madore, B. F., Scowcroft, V., et al. 2012, ApJ, 758, 24

Freeman, K., Ness, M., Wylie-de-Boer, E., et al. 2013, MNRAS, 428, 3660

Friedli, D., \& Benz, W. 1995, A\&A, 301, 649

Friel, E. D., Janes, K. A., Tavarez, M., et al. 2002, AJ, 124, 2693

Grevesse, N., Noels, A., \& Sauval, A. J. 1996, Cosmic Abundances, 99, 117

Groenewegen, M. A. T. 2013, A\&A, 550, A70

Groenewegen, M. A. T., Udalski, A., \& Bono, G. 2008, A\&A, 481, 441

Gustafsson, B., Edvardsson, B., Eriksson, K., et al. 2008, A\&A, 486, 951
Haschke, R., Grebel, E. K., \& Duffau, S. 2012, AJ, 144, 107

Hill, V., Lecureur, A., Gómez, A., et al. 2011, A\&A, 534, A80

Honma, M., \& Sofue, Y. 1997, PASJ, 49, 453

Inno, L., Matsunaga, N., Bono, G., et al. 2013, ApJ, 764, 84

Koen, C., Marang, F., Kilkenny, D., \& Jacobs, C. 2007, MNRAS, 380, 1433

Kim, S. S., Saitoh, T. R., Jeon, M., et al. 2011, ApJ, 735, L11

Kormendy, J., \& Kennicutt, R. C., Jr. 2004, ARA\&A, 42, 603

Kormendy, J., Fisher, D. B., Cornell, M. E., \& Bender, R. 2009, ApJS, 182, 216

Kovtyukh, V. V., \& Gorlova, N. I. 2000, A\&A, 358, 587

Kovtyukh, V. V., Wallerstein, G., \& Andrievsky, S. M. 2005, PASP, 117, 1173

Kraft, R. P., \& Schmidt, M. 1964, The Galaxy and the Magellanic Clouds, 20, 68

Kupka, F. G., Ryabchikova, T. A., Piskunov, N. E., Stempels, H. C., \& Weiss, W. W. 2000, Balt. Astron., 9, 590

Laney, C. D., \& Stobie, R. S. 1992, A\&AS, 93, 93

Leavitt, H. S., \& Pickering, E. C. 1912, Harvard College Observatory Circular, 173,1

Lemasle, B., François, P., Bono, G., et al. 2007, A\&A, 467, 283 (Paper I)

Lemasle, B., François, P., Piersimoni, A., et al. 2008, A\&A, 490, 613 (Paper III)

Lubowich, D. A., Pasachoff, J. M., Balonek, T. J., et al. 2000, Nature, 405, 1025

Luck, R. E., \& Lambert, D. L. 2011, AJ, 142, 136

Luck, R. E., Gieren, W. P., Andrievsky, S. M., et al. 2003, A\&A, 401, 939

Luck, R. E., Kovtyukh, V. V., \& Andrievsky, S. M. 2006, AJ, 132, 902

Luck, R. E., Andrievsky, S. M., Kovtyukh, V. V., Gieren, W., \& Graczyk, D. 2011, AJ, 142, 51

Martins, F., Hillier, D. J., Paumard, T., et al. 2008, A\&A, 478, 219

Matsunaga, N., Feast, M. W., \& Soszyński, I. 2011a, MNRAS, 413, 223

Matsunaga, N., Kawadu, T., Nishiyama, S., et al. 2011b, Nature, 477, 188

Matsunaga, N., Feast, M. W., Kawadu, T., et al. 2013, MNRAS, 429, 385

Matthews, L. D., Marengo, M., Evans, N. R., \& Bono, G. 2012, ApJ, 744, 53

Merlin, E., \& Chiosi, C. 2007, A\&A, 473, 733

Molinaro, R., Ripepi, V., Marconi, M., et al. 2011, MNRAS, 413, 942

Monson, A. J., \& Pierce, M. J., 2011, ApJS, 193, 12

Morris, M., \& Serabyn, E. 1996, ARA\&A, 34, 645

Najarro, F., Figer, D. F., Hillier, D. J., Geballe, T. R., \& Kudritzki, R. P. 2009, ApJ, 691, 1816

Neilson, H. R., Langer, N., Engle, S. G., Guinan, E., \& Izzard, R. 2012, ApJ, 760, L18

Ness, M., Freeman, K., Athanassoula, E., et al. 2012, ApJ, 756, 22

Ness, M., Freeman, K., Athanassoula, E., et al. 2013, MNRAS, 430, 836

Nishiyama, S. 2005, Astronomical Herald, 98, 240

Pedicelli, S., et al. 2009, A\&A, 504, 86 (Paper IV)

Pedicelli, S., Lemasle, B., Groenewegen, M., et al. 2010, A\&A, 518, A11 (Paper V)

Pietrzyński, G., Thompson, I. B., Gieren, W., et al. 2010, Nature, 468, 542

Portinari, L., \& Chiosi, C. 2000, A\&A, 355, 929

Prada Moroni, P. G., Gennaro, M., Bono, G., et al. 2012, ApJ, 749, 108

Reid, M. J., Menten, K. M., Zheng, X. W., Brunthaler, A., \& Xu, Y. 2009, ApJ, 705,1548

Romaniello, M., Primas, F., Mottini, M., et al. 2008, A\&A, 488, 731 (Paper II)

Samus, N. N., Durlevich, O. V., et al. 2009, VizieR Online Data Catalog, II/025

Skrutskie, M. F., Cutri, R. M., Stiening, R., et al. 2006, AJ, 131, 1163

Soszyński, I., Gieren, W., Pietrzyński, G. et al. 2005, PASP, 117, 823

Storm, J., Gieren, W., Fouqué, P., et al. 2011a, A\&A, 534, A94

Storm, J., Gieren, W., Fouqué, P., et al. 2011b, A\&A, 534, A95

Sziládi, K., Vinkó, J., Poretti, E., Szabados, L., \& Kun, M. 2007, A\&A, 473, 579

Twarog, B. A., Ashman, K. M., \& Anthony-Twarog, B. J. 1997, AJ, 114, 2556

Uttenthaler, S., Schultheis, M., Nataf, D. M., et al. 2012, A\&A, 546, A57

Wakker, B. P., Howk, J. C., Savage, B. D., et al. 1999, Nature, 402, 388

Yong, D., Carney, B. W., Teixera de Almeida, M. L., \& Pohl, B. L. 2006, AJ, 131,2256

Yusef-Zadeh, F., Hewitt, J. W., Arendt, R. G., et al. 2009, ApJ, 702, 178

Zánmar Sánchez, R., Sellwood, J. A., Weiner, B. J., \& Williams, T. B. 2008, ApJ, 674, 797

Zaritsky, D., Kennicutt, R. C., Jr., \& Huchra, J. P. 1994, ApJ, 420, 87

Zoccali, M., Renzini, A., Ortolani, S., et al. 2003, A\&A, 399, 931

Zoccali, M., Hill, V., Lecureur, A., et al. 2008, A\&A, 486, 177 